\title{
Effect of polymer molecular weight on adsorption and suspension rheology
}

\author{
Ungsoo KIM $^{\dagger}$ and William Michael CARTY* \\ Korea Institute of Ceramic Engineering \& Technology, Icheon, Kyonggido Korea 17303 \\ *New York State College of Ceramics at Alfred University, Alfred, NY USA 14802
}

\begin{abstract}
The adsorption of poly(acrylic acid) [PAA] on the surface of commercial ball clay was studied. The adsorption isotherm of PAAs with different molecular weights was determined at $\mathrm{pH} 6$ and 9. The results showed that the amount of PAA adsorbed on clay was independent of molecular weight. Also, the adsorption amount was decreased with increased $\mathrm{pH}$. However, the overall adsorption amount was lower than the range predicted by a model based upon the mineralogy of kaolinite. A kinetic study of PAA adsorption presented plausible displacement of initially adsorbed PAA. PAA adsorption on clay particles decreased the viscosity of suspensions. Clay suspensions showed the minimum viscosity with a sufficient coverage of particles with PAA necessary for stable suspensions. Optimum chain length of PAA was revealed for effective dispersion.
\end{abstract}

(2016 The Ceramic Society of Japan. All rights reserved.

Key-words : Polyelectrolyte, Molecular weight, Adsorption, Viscosity, Clay

[Received September 25, 2015; Accepted January 31, 2016]

\section{Introduction}

Clay is one of the major raw materials used in ceramic industries. Clay is known to serve a dual purpose; (1) provide good plasticity during forming process and (2) assist to generate a relatively viscous liquid essential to the densification during firing process. ${ }^{1)}$ It is necessary for both purposes to homogeneously distribute clay particles with other component materials. Failure to the homogenization leads to the forming defects and poor microstructure and mechanical property. In aqueous processing clay is usually mixed with other component materials in water and handled for the subsequent processes. Hence, the dispersion of clay particles with a proper dispersant in water is crucial for the homogenization. For this reason numerous studies have been performed to investigate the efficiency of dispersant on the clay particle stabilization. ${ }^{2-4)}$

Polyelectrolyte has been commonly used as a dispersant for clay particles in aqueous media, which can generate a repulsive interaction by electrostatic and steric effects. Most research efforts have been focused on the adsorption behavior of polyelectrolyte on clay correlating to the suspension stabilization. It has been found that the state of clay dispersion is affected by solution chemistry and polyelectrolyte since the interactive role between polymer, solvent, and particle surface determines the adsorption and polymer conformation at the interface. ${ }^{5)}$ This indicates that for a system with fixed variables of adsorbent surface and solution chemistry the property of polyelectrolyte has a decisive role for adsorption and stabilization.

Radius of gyration associated with molecular weight governs the number of charges accessible to the polyelectrolyte. ${ }^{6}$ Polymer adsorption is reported to generally increase with decreasing molecular weight. The relative importance of a pure electrostatic repulsion and a steric repulsion is closely related to the segment density profile at the interface. ${ }^{7)}$ The hydrodynamic layer thickness is independent of the molecular weight at low salt

\footnotetext{
Corresponding author: U. Kim; E-mail: ukim@kicet.re.kr
}

concentration, but increases with the molecular weight at high salt. ${ }^{8)}$ Despite of numerous studies on polymer adsorption and stabilization, description for electrosteric stabilization is still incomplete. Specially, there is a lack of systematic study in the relationship between the polymer property, adsorption, and dispersion. Molecular weight of polymer is one of the vital information for understanding the property of polymer such as solvency and adsorption energy. ${ }^{9}$ )

The objective of this study is to define the relationship between polymer property (molecular weight), adsorption, and suspension behavior. Sodium salt of polyacrylic acid with various molecular weights, which is one of most commonly used anionic polyelectrolytes for kaolinite dispersion, is chosen for this study. The adsorption isotherm of polyacrylic acid on commercial ball clay is measured at pH 6 and 9. Also, adsorption kinetics of PAA is studied. The effect of solution chemistry and molecular weight on the adsorption behavior is verified. Viscosity of suspensions is measured with the increase of PAA concentration. The suspension properties are correlated with polymer adsorption and configuration changes.

\section{Experimental procedures}

\subsection{Materials}

Huntingdon clay was kindly donated by United Clays (Brentwood, TN) for this investigation. Table 1 lists the chemical composition of the clay. The reported chemical analysis of the powders revealed relatively high amount of $\mathrm{TiO}_{2}$. Specific surface area was measured as $20 \mathrm{~m}^{2} / \mathrm{g}$ via Brunauer, Emmett, and Teller (BET) theory of nitrogen adsorption.

Sodium salts of polyacrylic acids having different molecular weights were provided by Rohm and Hass (Philadelphia, PA).

Table 1. Chemical Composition and Physical Properties of Huntingdon Clay

\begin{tabular}{cccccccccc}
\hline & $\mathrm{SiO}_{2}$ & $\mathrm{Al}_{2} \mathrm{O}_{3}$ & $\mathrm{Fe}_{2} \mathrm{O}_{3}$ & $\mathrm{TiO}_{2}$ & $\mathrm{CaO}$ & $\mathrm{MgO}$ & $\mathrm{Na}_{2} \mathrm{O}$ & $\mathrm{K}_{2} \mathrm{O}$ & LOI \\
\hline Wt. \% & 44.7 & 38.3 & 0.6 & 2.4 & 0.1 & 0.1 & 0.1 & 0.1 & 13.6 \\
\hline
\end{tabular}


Table 2. Reported Properties of Tested PAAs

\begin{tabular}{cccc}
\hline M.W. $(\mathrm{g} / \mathrm{mol})$ & PDI $^{*}$ & \% Solid & Sources \\
\hline 2000 & $1.27-1.3$ & 44 & Rohm \& Haas \\
3600 & $1.27-1.6$ & 42 & Rohm \& Haas \\
4500 & $1.27-1.3$ & 45 & Rohm \& Haas \\
10000 & $1.27-1.3$ & 40 & Rohm \& Haas \\
55000 & $1.27-1.3$ & 27 & Rohm \& Haas \\
\hline
\end{tabular}

* PDI: Polydispersity Index.

The properties of these polymers are listed in Table2.

\subsection{Methods}

The potentiometric titrations (ABU900, Radiometer, Cleveland, $\mathrm{OH}$ ) were performed on polymer solutions and a blank solution. In order to obtain $\mathrm{pH}$ titration curves for $\mathrm{pH} 2-10$ solution was titrated with $1 \mathrm{M} \mathrm{HCl}$ to $\mathrm{pH} 2$. One minute equilibration period was allowed between each addition of titrant. The suspension was then allowed to equilibrate for one hour, and then back-titrated with standardized $2 \mathrm{M} \mathrm{NaOH}$ (Fisher Scientific, Pittsburgh, PA). An identical titration was performed on a blank solution without polymers. The net uptake of hydroxyl ions as a function of $\mathrm{pH}$ was obtained by subtracting the blank titration curve from the sample curve.

Adsorption isotherms of PAA on clay particles were determined by the solution depletion method. ${ }^{10)}$ Suspensions at $15 \mathrm{v} / \mathrm{o}$ were prepared by mixing clay in de-ionized water with increasing polymer concentration from 0 to $0.6 \mathrm{mg} / \mathrm{m}^{2}$ based on clay surface area. Unit of $\mathrm{mg} / \mathrm{m}^{2}$ indicates the polymer weight in $\mathrm{mg}$ per the specific surface area of clay particles. The suspensions were adjusted to target $\mathrm{pH}$ values of 6 and 9 with tolerance of \pm 0.2 . The suspensions were mixed in a shaker bath for $24 \mathrm{~h}$. Suspensions were then centrifuged at $5000 \mathrm{rpm}$ for $60 \mathrm{~min}$, and the supernatant was removed without disturbing the sediment. The supernatants were adjusted to $\mathrm{pH} 10$ using $\mathrm{NaOH}$ and then titrated with $0.25 \mathrm{M} \mathrm{HCl}$ to $\mathrm{pH}$ 2. The amount of PAA in solution was determined by using calibration curves for PAA. The adsorbed amount was calculated as the difference between the added amount of polymer and the amount of polymer remaining in solution:

$$
\Gamma^{\mathrm{ex}}=\Delta \mathrm{C}_{\mathrm{p}} \mathrm{V} / \mathrm{A}_{\mathrm{s}}
$$

Adsorption kinetics of PAA on clay particles were studied by constructing adsorption isotherms at different adsorption times. Huntingdon clay and PAA with molecular weight of $2000 \mathrm{~g} / \mathrm{mol}$ were used for this study. Suspensions were prepared at two polymer concentration levels, 0.1 and $0.3 \mathrm{mg} / \mathrm{m}^{2}$ and adjusted to $\mathrm{pH}$ 9. Suspensions were kept on a shaker table from $6 \mathrm{~h}$ to 10 days. The adsorbed amount was determined as described above.

Suspensions were prepared at $35 \mathrm{v} / \mathrm{o}$ solids loading by mixing the Huntingdon clay in distilled water. PAA was first dissolved in water and equilibrated for $10 \mathrm{~min}$, and then clay was added slowly. Samples at different polymer concentrations from 0 to $1.0 \mathrm{mg} / \mathrm{m}^{2}$ were prepared. The prepared suspensions were placed on a shaker bath for 7 days to avoid problems occurring with aging. The $\mathrm{pH}$ of the suspension was not adjusted. The $\mathrm{pH}$ of the suspension without PAA was 4.08 and the highest $\mathrm{pH}$ with the addition of PAA was 6.8 .

Viscosity measurements were made using a strain-controlled rheometer (Advanced Rheometric Expansion System, Rheometric Scientific, Inc., Piscataway, NJ) and software (RSI Orchestrator Version V6.4.3, Rheometric Scientific, Inc., Piscataway, $\mathrm{NJ})$. A steady state stress sweep test from high to low rates with

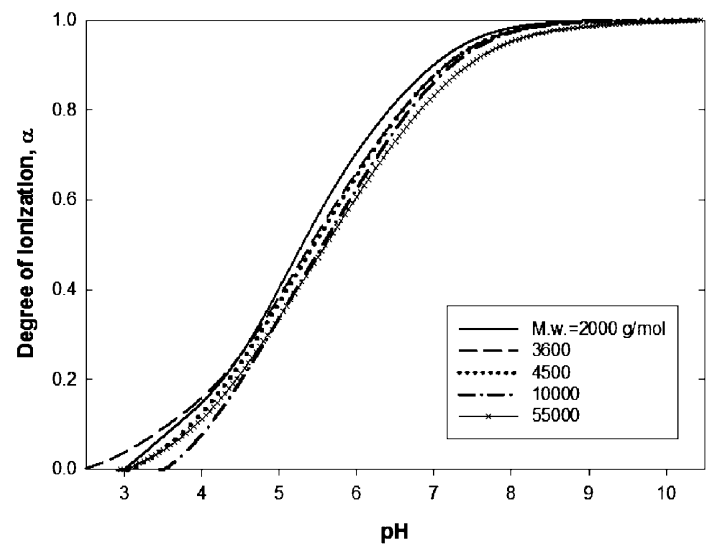

Fig. 1. Degree of ionization $(\alpha)$ of PAAs with $\mathrm{pH}$. Degree of ionization of PAA is essentially independent of molecular weight.

$25 \mathrm{~mm}$ parallel plates was used for all viscosity measurements. Each sample was measured for three times.

The $\zeta$-potentials were obtained through electroacoustic means (ESA-8000 AcoustoSizer, Matec Applied Sciences, Hopkinton, MA) on $5 \mathrm{v} / \mathrm{o}$ suspensions. All $\mathrm{pH}$ adjustments were made using the automatic titration system accompanying the AcoustoSizer.

\section{Results and discussions}

\subsection{Ionization of PAA}

The degree of ionization of PAA is calculated from the net uptake of hydroxyl ions and is shown as a function of $\mathrm{pH}$ in Fig. 1. It is known that the potentiometric titration curve of the PAA is essentially independent of molecular weight because the dissociation is determined by short range electrostatic interactions. ${ }^{11)}$ All the titration curves for PAA show similar trends and there is a slight increase in ionization with decreasing molecular weight in the intermediate degree of dissociation $(\alpha)$.

PAA starts to ionize around $\mathrm{pH} 3$ and is fully ionized at $\mathrm{pH} 10$. The most visible consequence of ionization is the solubility in water. $^{12)}$ The polymer charge becomes strong enough to overcome the chain hydrophobicity at high $\mathrm{pH}$, thereby making hydrophobic acids water soluble. Unlike low molecular weight acids the charged groups on polyacids are correlated because they are linked together along the chain. Therefore, as charge density on the chain varies, the chain conformation is affected and, in turn, influences the dissociation of other groups.

An important characteristic of polyelectrolytes is their structural transition as charge density increases along the flexible chain; polyelectrolyte molecules undergo expansion from a coiled to a stretched conformation. The polyelectrolyte chains are coiled due to van der Waals attractive forces and hydrogen bonding between segments, and the cooperative transition occurs due to electrostatic repulsion between neighboring ionized sites. $^{13)}$

\subsection{Adsorption kinetics of PAA (M.w. $=2000$ )}

The adsorption kinetics of PAA onto Huntingdon clay was determined at two polymer concentrations, 0.1 and $0.3 \mathrm{mg} / \mathrm{m}^{2}$. These two polymer additions correspond to the maximum amount of polymer adsorbed and the amount of polymer necessary to achieve the plateau region, respectively. The $\mathrm{pH}$ of all the suspensions was kept at $9.0 \pm 0.2$. Mixing time of suspensions was from six hours to ten days since there is a relatively large uncertainty for short mixing times due to the time needed for centrifugation. 


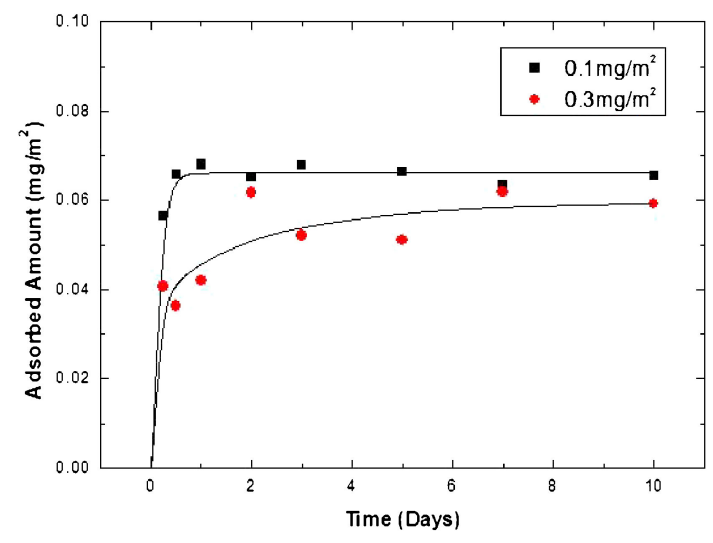

Fig. 2. Kinetics of PAA adsorption on Huntingdon clay. The adsorbed amount at low polymer concentration increases rapidly and reaches a plateau region.

Figure 2 shows the change in adsorption with time. The adsorbed amount at polymer concentration of $0.1 \mathrm{mg} / \mathrm{m}^{2}$ increases rapidly over $24 \mathrm{~h}$ and reaches a plateau region. For polymer concentration at $0.3 \mathrm{mg} / \mathrm{m}^{2}$ polymer adsorption seems to increase over the entire test period. The adsorbed amount at polymer concentration $0.3 \mathrm{mg} / \mathrm{m}^{2}$ is slightly lower than that for polymer concentration at $0.1 \mathrm{mg} / \mathrm{m}^{2}$.

The dependence of adsorption on time at different polymer concentrations may be affected by the displacement of initially adsorbed small polymer molecules by higher molecular weight ones. For polymer concentration of $0.1 \mathrm{mg} / \mathrm{m}^{2}$ most polyelectrolyte molecules adsorb on the surfaces and the number of longer molecules left in solution will be small. Accordingly, the displacement of small molecules on the surface with longer ones will be minimal. However, in the case of polymer concentration $0.3 \mathrm{mg} / \mathrm{m}^{2}$, there will be a relatively large number of longer molecules in solution. Therefore, an exchange process will occur with time.

It is noteworthy to explain the kinetic aspect of polyelectrolyte adsorption to understand the exchanging process in polyelectrolyte systems. When no electrostatic interaction is involved, an exchange process is caused by the diffusion and concentration of the chains in various lengths in solution. For polyelectrolytes the electrostatic barriers formed on particles by the adsorption of charged polyelectrolytes limit the size of long molecules displacing small ones. ${ }^{14)}$

The total charge of the PAA covered surface of the particles and the total charge on the chain in the solution determine the height of the electrostatic barriers. The charge on the chain is directly related to chain length and solution $\mathrm{pH}$. An exchange process will begin directly after initial adsorption occurs admitting preferential adsorption of the longest chains in thermodynamic equilibrium. However, the diffusion of polyelectrolytes towards the surface is affected by the electrostatic barrier; the longer the chains the less chance they have of reaching the surface. Polymers above a certain chain length will have too high of an electrostatic barrier and displacement of lower molecular weight will not occur. Also, the addition of salt facilitates the exchanging process by screening the charges. As the salt concentration increases, more short chains are displaced by longer ones. Therefore, in the system studied here an exchange process occurs and the process is accelerated with the dissolution of cations from clay.

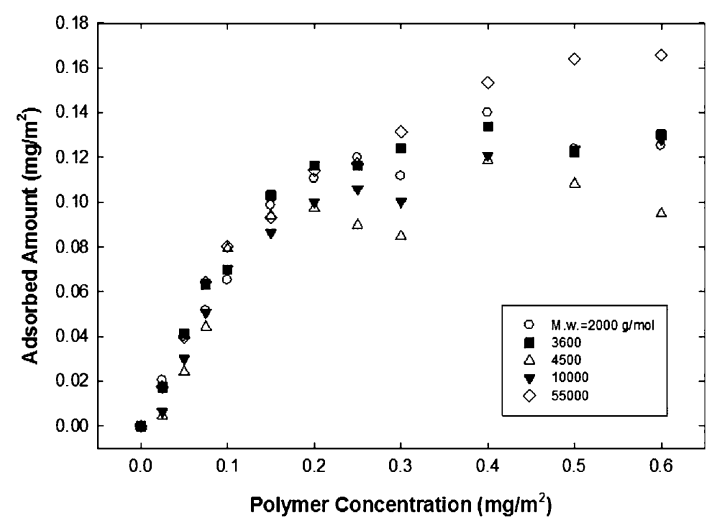

Fig. 3. Adsorption isotherm of various PAAs on Huntingdon clay at $\mathrm{pH}$ 6. Little effect of polymer molecular weight is seen on the adsorption plateau value.

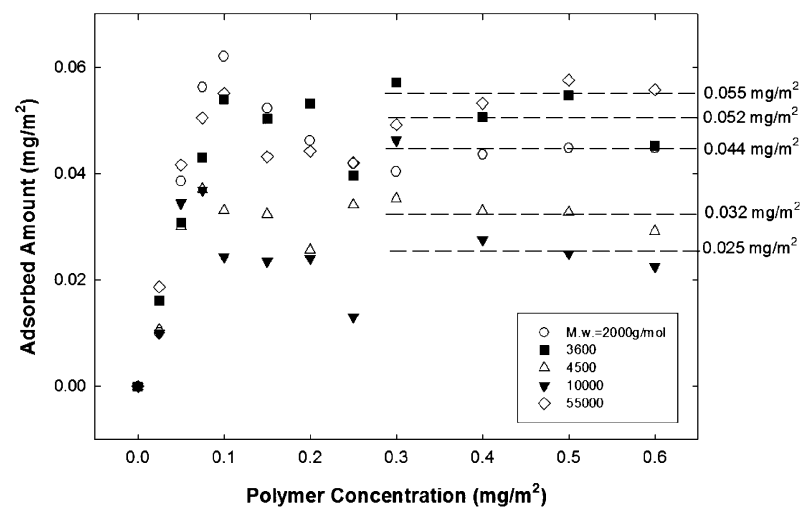

Fig. 4. Adsorption isotherm of various PAAs on Huntingdon clay at $\mathrm{pH}$ 9. The number next to the plot indicates the average adsorption amount.

\subsection{Effects of suspension $\mathrm{pH}$ and polymer molec- ular weight on adsorption}

The adsorption isotherms of PAA of different molecular weights were determined at $\mathrm{pH} 6$ and 9. Figures 3 and $\mathbf{4}$ show the adsorption behavior of PAA at $\mathrm{pH} 6$ and 9. The adsorption plateau for tested PAAs ranges from 0.02 to $0.06 \mathrm{mg} / \mathrm{m}^{2}$ at $\mathrm{pH} 9$, and from 0.1 to $0.16 \mathrm{mg} / \mathrm{m}^{2}$ at $\mathrm{pH}$. The dependence of adsorbed amount on the molecular weight of PAA is not shown.

A kaolinite particle is composed of a silica-like and an alumina-like surfaces with edges of a mixture of alumina and silica sites. The behavior of each surface in water is similar to silica and alumina, respectively. Polymers were presumed to primarily adsorb on the edge of the clay particles. However, Carty proposed that polymer adsorption occurs on a basal surface rather than the edge based on the relative adsorption limits and SEM analysis. ${ }^{15)}$ PAA also has a weak affinity for silica surfaces. Therefore, polymers will adsorb on the alumina-like surface as well as the edge of the clay platelet.

As $\mathrm{pH}$ increases, the net positive surface charge on aluminalike surface decreases while PAA is fully dissociated and negatively charged. Accordingly, adsorption of PAA decreases. As reported earlier, at $\mathrm{pH} 6$ the adsorbed polymer chains exhibit a coiled conformation. As the $\mathrm{pH}$ increases, the adsorbed PAA stretches out. ${ }^{16)}$ The stretching is less at the higher polymer concentration apparently due to crowding of the polymer chains on the particles at the higher surface coverage and resultant 
restricted expansion of the adsorbed coils.

The adsorption behavior of PAA on silica-like surface may be assumed from the PAA adsorption study on silica. Small amounts of PAA adsorb on silica in the acidic condition. However, PAA is completely desorbed from the surface with increasing negative surface charge and increasing dissociation. ${ }^{17)}$ Thus, in the suspensions tested here the adsorption of PAA on silica-like surface will be negligible.

The adsorbed amount was lower than expected at both $\mathrm{pH} 6$ and 9. Assuming the adsorption on one basal plane and the platelet edge, when normalized for the specific surface area of the powder, the adsorption limit for clay is expected to be $59 \%$ of the polymer adsorbed on an alumina surface. This value is based upon an aspect ratio of 0.095 (2L:h) for the clay platelet. PMAA has very similar chemical structure and adsorption behavior to PAA. The adsorbed PMAA amount on alumina particles was $\sim 0.35 \mathrm{mg} / \mathrm{m}^{2}$ at $\mathrm{pH} 9$ and $\sim 0.7 \mathrm{mg} / \mathrm{m}^{2}$ at $\mathrm{pH} 6{ }^{1{ }^{18)}}$ Therefore, expected adsorption on clay particles is $0.21 \mathrm{mg} / \mathrm{m}^{2}$ at $\mathrm{pH} 9$ and $0.41 \mathrm{mg} / \mathrm{m}^{2}$ at $\mathrm{pH}$ 6. However, the experimental value was much lower than the expected values. The discrepancy was greater at $\mathrm{pH} 9$.

At pHs 6 and 9 the PAA adsorption follows the same slope at low polymer concentrations, and the adsorption curve at $\mathrm{pH} 6$ goes further and reaches the plateau. The same slope may suggest the same adsorption affinity at both pHs. ${ }^{19)}$ The long rounded portion at $\mathrm{pH} 6$ may be related to the preferential adsorption. Vermeer and Koopal noted that the molecular weight dependence in the preferential adsorption increases with decreasing $\mathrm{pH}^{20)}$

At both $\mathrm{pH}$ values the adsorbed amount does not essentially depend on the molecular weight. Blaakmeer et al. developed adsorption model of weak polyacids at a charged surface in a low ionic strength medium. They expected a weak dependence on chain length at slightly higher $\mathrm{pH}$ values near $\mathrm{pK}_{0}$ value and no dependence at high $\mathrm{pH}$ values. ${ }^{21)}$ This result matches well with the experimental data in this study.

\subsection{Effect of polymer molecular weight on sus- pension rheology}

The steady shear properties of Huntingdon clay with PAA addition were determined. All the suspensions prepared with different molecular weights of PAA show shear thinning behavior in which viscosity decreases with shear rate. Figure 5 shows the dependence of the viscosity on the shear rate with the addition of PAA (m.w. $=2000)$ for an example.

The decrease in viscosity and degree of shear thinning with the

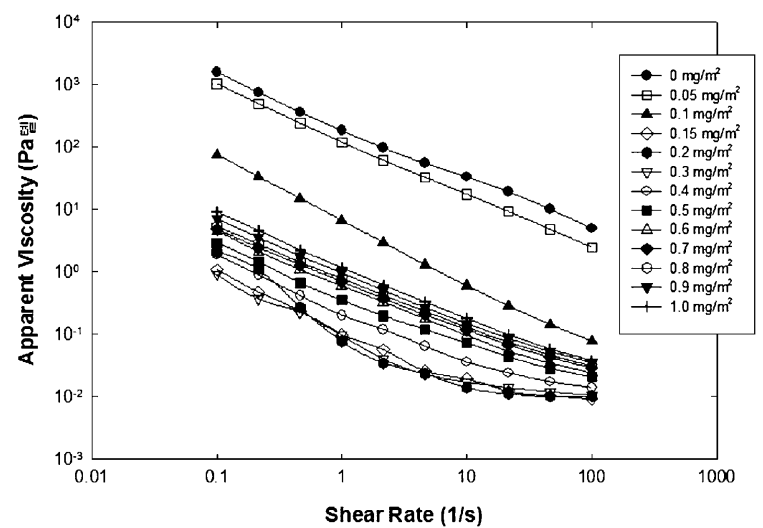

Fig. 5. Apparent viscosity versus shear rate of Huntingdon clay with the addition of PAA (m.w. = $2000 \mathrm{~g} / \mathrm{mol})$. Viscosity decreases with shear rate for all polymer concentrations tested. addition of PAA implies a decrease in the degree of flocculation. The charged PAA adsorbs on the oppositely charged surface sites of clay particles and exhibits short-range repulsion. Both steric and electrostatic forces may contribute to the overall repulsion. The charge density and the conformation of PAA are altered by adjusting the solution conditions. ${ }^{22}$ ) Under acidic conditions, where all the tested suspensions were prepared, ionization of PAA is relatively small and PAA forms a compact coil. ${ }^{23)}$

Around $0.2 \mathrm{mg} / \mathrm{m}^{2}$ PAA concentration most suspensions reach the minimum viscosity. Figure 6 shows that the zeta potential reaches the plateau region around $0.2-0.3 \mathrm{mg} / \mathrm{m}^{2}$ PAA addition. The adsorption isotherm at $\mathrm{pH} 6$ also indicates that plateau is reached around this polymer concentration. This suggests that electrostatic repulsion by adsorbed polyelectrolyte is high enough to overcome attractive forces. In addition, the adsorbed polymer layer on the surface is strong and sufficiently thick with high surface coverage, thus, steric repulsion occurs

With further addition of PAA the viscosity of suspensions increases, this reflects a transition from stabilization to flocculation. For long chain polymers bridging is most likely to happen. When non-adsorbing polymers exist in the suspension, depletion flocculation could arise as the particles approach and the polymers are excluded from the gap due to an osmotic pressure gradient between the particles. ${ }^{22}$ Other possibilities could be due to an increase in ionic strength, which may weaken the electrostatic repulsion, and/or the increase in the medium viscosity due to the increase of the free polymer. At higher dispersant levels the dispersants themselves contribute enough ions to the suspension to achieve the critical coagulation concentration. ${ }^{24)}$

The apparent viscosity was obtained by extrapolating to the shear rate at $1.0 \mathrm{~s}^{-1}$. Figure 7 shows the apparent viscosity of suspensions with the addition of PAA. All the suspensions with PAAs show the minimum viscosity at $0.2 \mathrm{mg} / \mathrm{m}^{2}$. The minimum viscosity was plotted versus molecular weight in Fig. 8. The lowest viscosity was obtained at molecular weight of 10000 $\mathrm{g} / \mathrm{mol}$. This suggests an optimum chain length of PAA for effective dispersion. The increase in viscosity at higher polymer concentrations is dependent on the molecular weight of the polymers. PAA of the highest molecular weight (m.w. $=55000$ $\mathrm{g} / \mathrm{mol}$ ) shows the lowest dispersability.

\section{Conclusions}

This study confirms the relationship between polymer adsorption and suspension rheology. The adsorption of PAA on clay particles decreases with increase in $\mathrm{pH}$. The surface charge of the

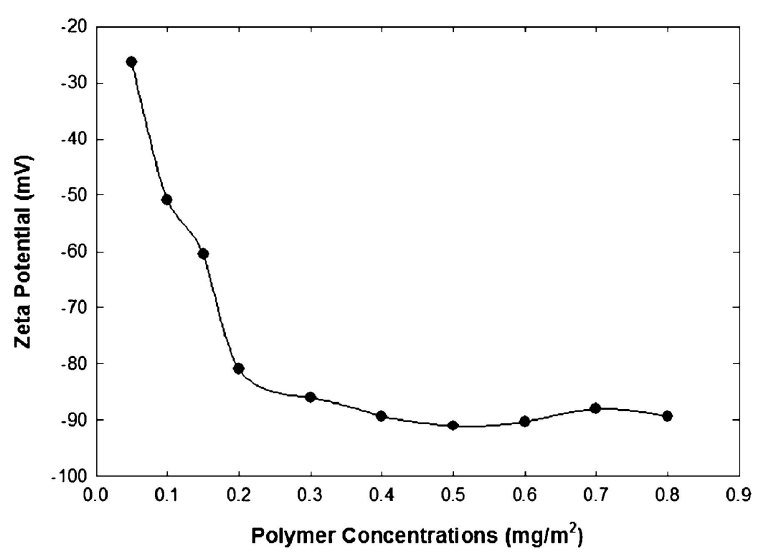

Fig. 6. Zeta potential of Huntingdon clay with the addition of PAA. Zeta potential reaches the plateau region around $0.3 \mathrm{mg} / \mathrm{m}^{2}$ PAA addition. 


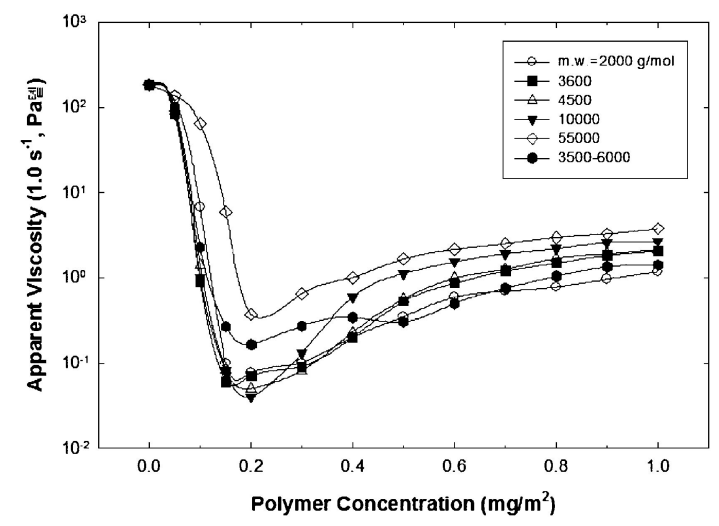

Fig. 7. Apparent viscosity change with the addition of PAAs. All the suspensions show the minimum viscosity approximately at $0.2 \mathrm{mg} / \mathrm{m}^{2}$ PAA addition. $\mathrm{pH}$ was not adjusted in these suspensions.

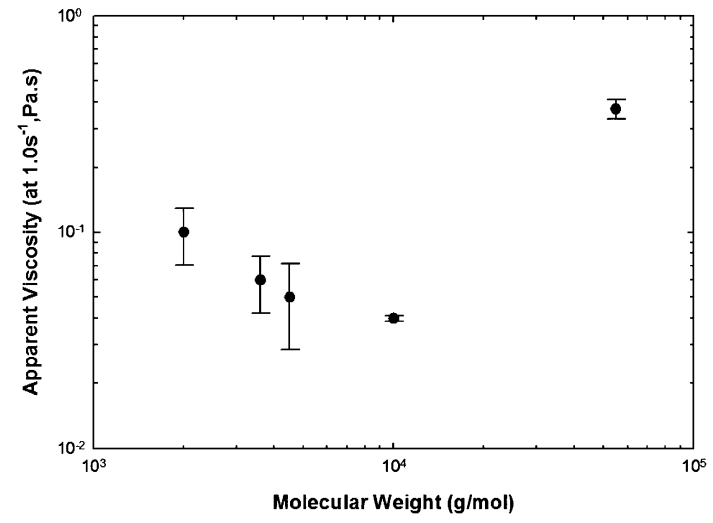

Fig. 8. Apparent viscosity versus molecular weight of PAAs. The lowest viscosity is obtained at molecular weight of $10000 \mathrm{~g} / \mathrm{mol}$.

clay particle and the polymer conformation change due to polymer charge explain the adsorption behaviors of PAA on clay particles. However, it is shown that organic matter in clay seems to interfere the adsorption of PAA and results in lower adsorption amount. The PAA adsorption amount is not influenced by the molecular weight of PAA. A kinetic study of PAA adsorption reveals that the polymer adsorption is influenced by the displacement of initially adsorbed PAA.

PAA adsorption on clay particles decreases the viscosity of suspensions. Clay suspensions show the minimum viscosity at a certain amount of PAA addition, which suggests that electrostatic force and sufficient coverage of particles with PAA are necessary for stable suspensions. Also, there is an optimum chain length of PAA for effective dispersion.

\section{References}

1) W. D. Kingery, H. K. Bowen and D. R. Uhlmann, Introduction to Ceramics. 2nd ed. New York: Wiley-Interscience (1975).

2) A. B. Corradi, T. Manfredini, G. C. Pellacani and P. Pozzi, J. Am. Ceram. Soc., 77, 509-513 (1994).

3) B. Rand and I. E. Melton, J. Colloid Interface Sci., 60, 308320 (1977)

4) T. Manfredini, G. C. Pellacani, P. Pozzi and A. B. Corradi, Appl. Clay Sci., 5, 193-201 (1990).

5) J. Cesarano, Polyelectrolyte Adsorption in $\alpha$-Alumina and Aqueous Suspension Behavior [dissertation]. Seattle (WA): University of Washington (1986).

6) M. Gimaker, Influence of adsorbed Polyelectrolytes and Adsorption Conditions on Creep Properties of Paper Sheets made from Unbleached Kraft Pulp [dissertation]. Stockholm (Sweden): Royal Institute of Technology (2007).

7) H. Guldberg-Pedersen and L. Bergstrom, Acta Mater., 48, 4563-4570 (2000).

8) E. Seyrek, J. Hierrezuelo, A. Sadeghpour, I. Szilagyi and M. Borkovec, Phys. Chem. Chem. Phys., 13, 12716-12719 (2011).

9) H. Allcock and F. W. Lampe, Contemporary Polymer Chemistry. 2nd ed. New Jersey; Prentice Hall (1990).

10) G. J. Fleer, M. A. Cohen Stuart, J. M. H. M. Scheutjens, T. Cosgrove and B. Vincent, Polymers at Interfaces. London: Chapman \& Hall (1998).

11) S. Kawaguchi, T. Takahashi, H. Tajima, Y. Hirose and K. Ito, Polym. J. (Tokyo, Jpn.), 28, 735-741 (1996).

12) I. Borukhov, D. Andelman, R. Borrega, M. Cloitre, L. Leibler and M. Orland, J. Phys. Chem. B, 104, 11027-11034 (2000).

13) M. Sakurai, T. Imai, F. Yamashita, K. Nakamura and T. Komatsu, Polym. J., 25, 1247-1255 (1993).

14) A. W. M. de Laat and G. L. T. van den Heuvel, Colloid Surface A, 98, 53-59 (1995).

15) W. M. Carty, Am. Ceram. Soc. Bull., 78, 72-76 (1999).

16) Z. Pan, A. Campbell and P. Somasundaran, Colloid Surface A, 191, 71-78 (2001).

17) G. R. Jopplen, J. Phys. Chem., 82, 2210-2215 (1978).

18) J. Cesarano, I. A. Aksay and A. Bleir, Polyelectrolyte. J. Am. Ceram. Soc., 71, 250-255 (1988).

19) L. Guo, Y. Zhang, N. Uchida and K. Uematsu, J. Am. Ceram. Soc., 81, 549-556 (1998).

20) A. W. P. Vermeer and L. K. Koopal, Langmuir, 14, 4210-4216 (1998).

21) J. Blaakmeer, M. R. Bohmer, M. A. Cohen Stuart and G. J. Fleer, Macromolecules, 23, 2301-2309 (1990).

22) Z. Zhou, P. J. Scales and D. V. Boger, Chem. Eng. Sci., 56, 2901-2920 (2001).

23) Y. K. Leong, P. J. Scales, T. W. Healy and D. V. Boger, Colloid Surface A, 95, 43-52 (1995).

24) B. R. Sundlof, Aqueous Processing of Alumina and Phase Behavior of Polymeric Additives [dissertation]. Alfred (NY): Alfred University (1999). 